\title{
Prevalence of ESBLs among Enterobacteriaceae and their Antibiotic Resistance Pattern from Various Clinical Samples
}

\author{
Sanjo Gupta and Veena Maheshwari* \\ Department of Microbiology RKDFMCH \& RC Jatkhedi Bhopal, India \\ *Corresponding author
}

\begin{tabular}{|c|c|}
\hline & A B S T RACT \\
\hline & \multirow{10}{*}{$\begin{array}{l}\text { Beta-lactam antibiotics are the most commonly used antimicrobials against bacterial } \\
\text { infections. In recent years, emergence of resistance to these antimicrobial agents due to } \\
\text { production of } \beta \text {-lactamases has become a serious global health concern. It was reported as } \\
\text { leading cause to ineffectiveness to antibiotic usage, increased morbidity and high cost of } \\
\text { treatment. Extended spectrum beta-lactamases (ESBLs) detection amongst the Gram - } \\
\text { negative bacilli is considered as an important marker of endemicity. Knowledge of the } \\
\text { patterns of antimicrobial resistance and effective surveillance has significant implications } \\
\text { for patient management and guiding clinicians to take appropriate interventions. A } \\
\text { prospective study was conducted over a duration of } 1 \text { year (March } 2014 \text { to February 2015) } \\
\text { in the Department of Microbiology, of a teaching tertiary care hospital. The prevalence of } \\
\text { potential ESBLs producers was explored. Antimicrobial susceptibility was determined by } \\
\text { the Kirby-Bauer disc diffusion method according to Clinical Laboratory Standard Institute } \\
\text { (CLSI) guidelines. A total of } 250 \text { samples from male and female patients visiting the } \\
\text { outpatient department (OPD) and inpatient department (IPD) of our hospital were } \\
\text { collected. Among the ESBL producers maximum number of isolates were identified as E. } \\
\text { coli } 45(44.1 \%) \text { followed by Klebsiella spp. } 30(29.4 \%) \text {, Citrobacter spp. } 10(9.8 \%) \text { Proteus } \\
\text { spp. } 12(11.7 \%) \text { and Enterobacter spp. } 5(4.9 \%) \text { respectively. ESBLs producing isolates } \\
\text { were found to be multi-drug resistant when compared to non-ESBL producers. High } \\
\text { prevalence of ESBLs producing Enterobacteriaceae in hospitals, with a tendency for multi- } \\
\text { drug resistance, suggests that routine detection is mandatory as this may help in regulating } \\
\text { hospital antibiotic policy. }\end{array}$} \\
\hline & \\
\hline & \\
\hline & \\
\hline & \\
\hline Article Info & \\
\hline & \\
\hline & \\
\hline & \\
\hline & \\
\hline
\end{tabular}

\section{Introduction}

Beta-lactam antibiotics are the most commonly used antimicrobials against bacterial infections. In recent years, emergence of resistance to these antimicrobial agents due to production of $\beta$-lactamases has become a serious global health concern. It leads to antibiotic ineffectiveness, increased severity of illness and cost of treatment. ${ }^{1}$ these enzymes are numerous and they mutate continuously in response to overuse or misuse of beta-lactam antibiotics and have lead to the development of extended spectrum $\beta$ lactamases $(\mathrm{ESBL})^{2}$
Extended spectrum beta-lactamase (ESBL) producing organisms are those that hydrolyze the oxyimino beta-lactams and monobactams, but have no effect on the cephamycins and carbapenems and are commonly inhibited by $\beta$ - lactamase inhibitors such as clavulinic acid, sulbactam, and Tazobactam. ${ }^{3}$

ESBL are placed under Bush's functional class 2be. They are plasmid-mediated enzymes and are derived from point mutation of TEM on SHV $\beta$-lactamases that are widely distributed among the Enterobacteriaceae. In 
recent years, several new ESBLs of the nonTEM and the non-SHV types emerged, such as the enzymes of the CTX-M, PER, VEB, and the GES lineages. ${ }^{2}$

Risk factors for infection with ESBL producing organisms are prolonged antibiotic usage, ICU stay, recent invasive procedure, pressure sores, anaemia and permanent urinary catheter. ${ }^{4}$

The prevalence of these organisms varies geographically and in hospital settings. ESBLs have been reported worldwide in many different genera of Enterobacteriaceae and Pseudomonas aeruginosa ${ }^{5}$. However, majority of the ESBLs are found in Klebsiella spp. and Escherichia coli of the Enterobacteriaceae family. ${ }^{6,7}$ The serious increase in the prevalence of ESBL's worldwide creates a need for effective and easy to perform screening methods for detection.

ESBL producing isolates remain undetected as they are difficult to detect by routine susceptibility testing methods and may show false susceptibility to antibiotics by KirbyBauer disc diffusion methods. ${ }^{4}$

Several methods have been developed to detect the presence of ESBL including Double disc synergy test (DDST) and doubledisc diffusion test (DDDT) using cefotaxime and ceftazidime, disc with or without clavulanic acid. ${ }^{9}$

Although various studies have been conducted on the ESBL-producing strains of Enterobacteriaceae in different regions of India like Bhopal, ${ }^{2}$ Dibrugarh, ${ }^{10}$ Bangalore, ${ }^{11}$ Pondicherry, ${ }^{12}$ Bijapur, ${ }^{13}$ Hyderabad, ${ }^{14}$ Mumbai, ${ }^{15}$ no published data are available on the prevalence of ESBL production in the Moradabad region of Uttar Pradesh in Northern India. ESBL detection is of utmost importance to formulate infection control measures and to prevent their spread

Hence the present study was undertaken to find the prevalence and resistance pattern of ESBL producing organisms and to help in implementing an effective antibiotic policy.

\section{Materials and Methods}

A prospective study was conducted over a duration of 1 year (March 2014 to February 2015) in the Department of Microbiology, of a teaching tertiary care hospital. The study protocol was approved by the Institutional Ethical Committee and informed consent was obtained from patients.

A total of 250 samples from different clinical specimens such as urine, tracheal aspirate, pus, blood and sputum were collected from patients of all age groups and either sex in sterile containers.

Samples were processed and isolates were identified by standard laboratory methods. ${ }^{16,17}$ Antimicrobial susceptibility was determined by the Kirby-Bauer disc diffusion method according to Clinical Laboratory Standard Institute (CLSI) guidelines. ${ }^{18}$

The following antibiotics were tested: Amikacin (30 mcg), gentamycin $(10 \mathrm{mcg})$, ampicillin (10 $\mathrm{mcg})$, ampicillin/sulbactam (10/10 mcg), ciprofloxacin(5 mcg), levofloxacin (5 mcg), chloramphenicol (30 mcg), co-trimoxazole $(1.25 / 23.75 \mathrm{mcg})$, ceftriaxone $(30 \mathrm{mcg})$, cefotaxime $(30 \mathrm{mcg})$, ceftazidime $(30 \mathrm{mcg})$, tigecycline $(30 \mathrm{mcg})$ piperacillin-tazobactum $\quad(100 / 10 \quad \mathrm{mcg})$, imipenem $(10 \mathrm{mcg})$ and meropenem (10 $\mathrm{mcg})$,ertapenem(10 mcg). Norfloxacin (10 $\mathrm{mcg})$ and nitrofurantoin (300 $\mathrm{mcg})$ were tested against isolates from urine samples only. Dehydrated media and antibiotic discs were procured from Hi-Media, Mumbai. 
All isolates were subjected for ESBL screening test. Potential ESBL producer was then subjected for ESBL Phenotypic confirmatory test -Disc Diffusion method (PCDDT).

\section{Method for ESBL detection}

ESBL production was detected by using the phenotypic confirmatory test along with routine antibiotic susceptibility testing according to Clinical Laboratory Standard Institute (CLSI) guidelines18. Cefotaxime and ceftazidime discs alone and in combination with clavulinic acid were used.A $>5 \mathrm{~mm}$ increase in zone size was confirmed for ESBL production. $^{18}$

Although CLSI described phenotypic confirmatory test is applicable for Escherichia coli, Klebsiella pneumoniae, and Proteus mirabilis, an attempt was made to look for ESBL production among the other members of Enterobacteriaceae.

ESBL-producing Enterobacteriaceae isolates which were resistant to cefoxitin (zone diameter $<18 \mathrm{~mm}$ ) 18 were not considered for the study. This is to exclude associated AmpC type of $\beta$-lactamases. ${ }^{19,20}$

In the study, K. pneumoniae ATCC 700603 and E. coli ATCC 25922 (HiMedia Laboratories, Mumbai) were used as positive and negative controls throughout, for ESBL production

\section{Results and Discussion}

A total of 250 samples were collected from patients who consisted of $60(40 \%)$ males and $90(60 \%)$ females' patients visiting the outpatient departments (OPD) and inpatient departments (IPD). These included Urine, pus, swabs, Blood, sputum and tracheal aspirate. Most of the patients presented in the active age group of more than 21-30 years.
Prevalence of ESBL producers among male patients was 33(32.3\%) and female patients was $69(67.6 \%)$ respectively. Among the 150 Gram negative isolates $102(68 \%)$ were found to be ESBL producers and rest $48(32 \%)$ were non-ESBL producers (Table 1).

The age group more commonly affected was between 21 and 30 years $(n=38)$ (Table 2).

Among the ESBL producers maximum number of isolates were identified as $E$. coli 45(44.1\%) followed by Klebsiella spp. 30(29.4\%), Citrobacter spp. 10(9.8\%) Proteus spp. 12(11.7\%), and Enterobacter spp. 5(4.9\%) respectively.

And Amongst the Non ESBL producers highest percentage of isolates identified were E. coli $15(31.25 \%)$ followed by Klebsiella spp. 12(25\%), Citrobacter spp. 10(20.8\%) Proteus spp.6 (12.5\%) and Enterobacter spp. $5(10.4 \%)$ respectively.

Prevalence of ESBLs was maximum in ICU $34.2 \%$ and least in ENT 3.9\% (Table 3).

A total of 150 non repetitive clinical isolates of Enterobacteriaceae were identified, among which 60 (40\%) isolates were Escherichia coli, followed by $42(28 \%)$ isolates of Klebsiella spp., 20 (13.3) of Citrobacter spp $18(12 \%)$ of Proteus spp., and10 (6.6\%)of Enterobacter spp., respectively. The distribution of organisms isolated from the various clinical specimens is as shown in table 4 .

Infections by ESBL producing organisms have emerged as a major problem and the failure of therapy with broad spectrum antibiotics are creating serious problems. ${ }^{21}$

Although some studies report male sex to be a risk factor for ESBL production ${ }^{22,23}$ our study corroborates with the study conducted by Nibedita Das et al., where there was no 
significant association between ESBL production and male sex ${ }^{10}$. The present study revealed a slight female preponderance for ESBL production among the study subjects. This was similar to the findings of an earlier study which were reported by Kiratisin et al., ${ }^{24}$ which revealed a female preponderance.

In our present study, we isolated 102(68\%) ESBL producers and 48(32\%) were nonESBL producers. Their findings were quite different to several studies worldwide reflect the general trend in prevalence of ESBL producing bacteria. A study reported by Nibedita das et al., ${ }^{\mathbf{1 0}}$ from South India on ESBL production in uropathogens showed 81.9\% ESBL producers.

Out of the 150 Enterobacteriaceae isolates, a majority were E. coli (40\%), followed by Klebsiella pneumoniae (28\%), Citrobacter spp (13.3\%), Proteus spp (12\%),
Enterobacter spp (6.6\%). This finding was on par with those of many studies ${ }^{25}$. Mathur $e t$ al. ${ }^{26}$ from New Delhi, have also reported $E$. coli and Klebsiella pneumoniae as the most common Enterobacteriaceae which were prevalent in their clinical samples and this was well comparable to the reports from our study. Babypadmini et al., ${ }^{27}$ from Chennai too reported the prevalence of $49 \%$ E. coli and $8 \%$ Klebsiella spp.

Antibiotic resistance has been noted as a serious problem, even at our medical college hospital. The third generation cephalosporins have been used in a majority of patients and resistance even to these antibiotics has been reported. As there was no data which was available on the prevalence of ESBL production in this region, the current study was undertaken to know the prevalence of ESBL producing Enterobacteriaceae at our tertiary health care centre.

Table.1 Distribution of ESBL strains among male and female patients

\begin{tabular}{|l|l|l|l|}
\hline Sex & $\begin{array}{l}\text { ESBL } \\
\mathbf{N = 1 0 2}(\boldsymbol{\%})\end{array}$ & $\begin{array}{l}\text { NON-ESBL } \\
\mathbf{N = 4 8}\end{array}$ & TOTAL \\
\hline MALE & $33(32.35 \%)$ & $27(56.25 \%)$ & 60 \\
\hline FEMALE & $69(67.64 \%)$ & $21(43.75 \%)$ & 90 \\
\hline TOTAL & 102 & 48 & 150 \\
\hline
\end{tabular}

Table.2 Age-wise distribution of ESBL producing Enterobacteriaceae in different age groups

\begin{tabular}{|l|c|c|}
\hline Age interval & Males & Females \\
\hline $0-10$ & 0 & 1 \\
\hline $11-20$ & 3 & 5 \\
\hline $21-30$ & 10 & 28 \\
\hline $31-40$ & 8 & 12 \\
\hline $41-50$ & 5 & 8 \\
\hline $51-60$ & 4 & 9 \\
\hline $61-70$ & 2 & 4 \\
\hline $71-80$ & 1 & 2 \\
\hline
\end{tabular}


Table.3 Department wise distribution of ESBL producers

\begin{tabular}{|l|c|c|c|}
\hline Wards & No. of isolates & ESBL producer & Percentage (\%) \\
\hline Medicine & 25 & 17 & 16.6 \\
\hline Surgery & 25 & 15 & 14.7 \\
\hline Orthopedics & 20 & 14 & 13.7 \\
\hline OBG & 15 & 11 & 10.7 \\
\hline ENT & 9 & 4 & 3.9 \\
\hline Medical ICU & 17 & 13 & 12.7 \\
\hline Surgical ICU & 20 & 15 & 14.7 \\
\hline Pediatric & 10 & 6 & 5.8 \\
\hline ICCU & 9 & 7 & 6.8 \\
\hline Total & 150 & 102 & 68 \\
\hline
\end{tabular}

Table.4 Distribution of organisms isolated by specimen $(\mathrm{N}=150)$

\begin{tabular}{|l|l|l|l|l|l|l|l|}
\hline Organisms & Urine & Pus & Swab & Sputum & Blood & Tracheal aspirate & Total \\
\hline Escherichia coli & 42 & 10 & 9 & 5 & 4 & 2 & 60 \\
\hline Klebsiella spp. & 17 & 9 & 8 & 5 & 4 & 2 & 42 \\
\hline Citrobacter spp. & 9 & 2 & 3 & 4 & 2 & 0 & 20 \\
\hline Proteus spp. & 7 & 3 & 2 & 2 & 3 & 1 & 18 \\
\hline Enterobacter spp. & 3 & 2 & 1 & 2 & 1 & 1 & 10 \\
\hline Total & 78 & 26 & 23 & 18 & 10 & 6 & 150 \\
\hline
\end{tabular}

Fig.1 Graphical representation of the antibiotic resistance profile of various extended spectrum beta-lactamase (ESBL) and non-ESBL clinical isolates

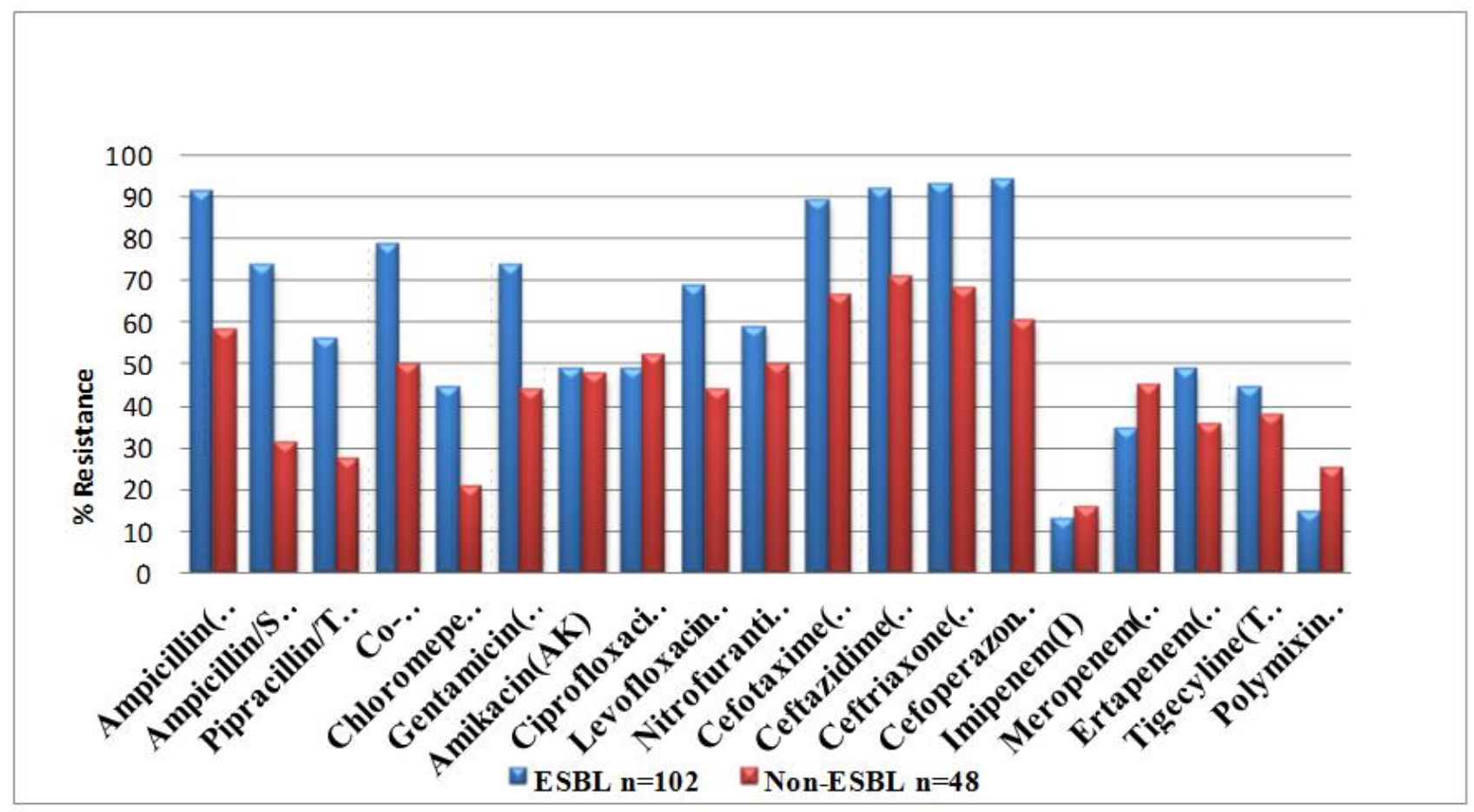


As of now, no countrywide study has been conducted for the detection of the prevalence of ESBL production in India. Individual studies which were done in different parts of the country showed a varying prevalence, based on various risk factors and local reasons.

In the present study, the highest number of ESBL producers were obtained in the isolates from the ICU $(\mathrm{N}=35,34.31 \%)$, the followed by Medicine $(\mathrm{N}=17,16.6 \%)$ and Surgery $(\mathrm{N}=$ $15,14.7 \%)$. This was comparable with a study which was carried out at AIIMS, New Delhi, India. ${ }^{27}$ this could be due to the prolonged hospital stay, inappropriate therapy, total antibiotic use, indwelling catheters, endotracheal or nasogastric tubes, gastrostomies or tracheostomies and the severity of the illness.

The antibiogram of the ESBL producing isolates showed a high degree of resistance towards routinely prescribed antibiotics when compared to non-ESBL producing isolates. These findings are similar to those reported by Sasirekha et al., Ndugulile $e t$ al., and Mehrgan et al., ${ }^{28,29,30}$ Since ESBLs are plasmid-mediated enzymes, which can be transferred from one bacterium to another and as these transferable plasmids also code for resistance determinants to antimicrobial agents other than beta-lactams. This finding is further explained by such fact. ${ }^{31}$

In our study, Resistance shown to piperacillin-tazobactum $\quad(55.8 \%$ and $27 \%$ resistance among ESBL and non-ESBL's) was low, reflects their less use for treatment of community-acquired infections. Not only ESBL producing isolates (resistance towards cefotaxime, ceftazidime, ceftriaxone and cefoparazone sulbactum in the range of 8994\%) but also ESBL nonproducers (cefotaxime: 66.7\%; ceftazidime: 70.7\%; ceftraxone 68.2\%; Cefoparazone sulbactum:
$60.1 \%$ ) possessed high degrees of resistance towards 3rd and 4th generation cephalosporins and mono-bactams. Such observation can be attributed to overuse of antibiotics in both community and hospital set- up, uncontrolled practices in prescription writing and incomplete dose consumed by patients.

Drugs such as imipenem which is used as last resort in the health-care settings was found resistant $(12.7 \%$ and $15.8 \%$ among ESBL producers and non-producers, respectively). In addition, drugs such as meropenem exhibited high degree of resistance (reported as $34.3 \%$ and $45 \%$ by ESBL and non-ESBL producers respectively) which is quite alarming. Resistance against carbapenem can be attributed to multifactorial causes. The mechanism through which it occurs is either bacterial production of beta-lactamases, that hydrolyze the antibacterial agent or through changes in porin channels in the bacterial cell wall that decrease the permeability of the drug into the organism. In addition, upregulation of efflux pumps result into reduced susceptibility of organisms toward meropenem. ${ }^{32}$ Most studies showed $100 \%$ sensitivity toward imipenem. ${ }^{33,22,34}$ Incidences of meropenem resistance higher than that of imipenem among nosocomial pathogens was observed by Gupta et al., ${ }^{35}$ The resistance exhibited in our case is due to existence of carbapenemase producing isolates in our setting. This may be because patients in Intensive Care Unit are directly being treated with carbapenems that has led to development of such multidrugresistant isolates in our health-care setting.

The detailed insight of antibiotic resistance pattern has been illustrated in figure 1 .

The present study highlights that there is a high prevalence of ESBL producing bacteria among Enterobacteriaceae mostly E. coli and Klebsiella spp. In view of these findings, we 
suggest that routine screening of ESBL should be performed on all isolates which are showing decreased susceptibility to third generation cephalosporins. The strict compliance to antibiotic stewardship and enforcement of infection control practices should also be strengthened in all our tertiary health centers.

\section{References}

1. Yazdi M, Nazemi A, Mirinargasi M, Jafarpour M, Sharifi SH. Genotypic versus Phenotypic methods to detect extendedspectrum beta-lactamases (ESBLs) in uropathogenic Escherichia coli. Ann Biol Res 2012;3:2454-8.

2. Shashwati N, Kiran T, Dhanvijay AG. Study of extended spectrum $\beta$-lactamase producing Enterobacteriaceae and antibiotic coresistance in a tertiary care teaching hospital. J Nat Sc BiolMed 2014;5:30-5.

3. Bajpai T, Pandey M, Varma M, Bhatambare GS. Prevalence of extended spectrum beta-lactamase producing uropathogens and their antibiotic resistance profile in patients visiting a tertiary care hospital in central India: Implications on empiric therapy. Indian J Pathol Microbiol 2014;57:407-12.

4. Singh N et al.Prevalence of ESBL in Escherichia coli Isolates Among ICU Patients in a Tertiary Care Hospital. Journal of Clinical and Diagnostic Research. 2016, Vol-10(9): DC19-DC22

5. Falagas ME, Karageorgopoulos DE. Extended spectrum beta lactamase producing organisms. $J$ Hosp Infect. 2009;73(4):345-54.

6. Mehrgan H, Rahbar M, Arab-Halvaii Z. High prevalence of extended-spectrum beta-lactamase-producing Klebsiella pneumoniae in a tertiary care hospital in Tehran, Iran. The Journal of Infection in Developing Countries. 2010;4(3):132-38.
7. Kassakian SZ, Mermel LA. Changing epidemiology of infections due to extended spectrum beta-lactamase producing bacteria. Antimicrobial Resistance and Infection Control. 2014;3(1):9.

8. Naiemi NA, Murk JL, Savelkoul PH, Vandenbroucke-Grauls CM, DebetsOssenkopp YJ. Extended-spectrum betalactamases screening agar with AmpC inhibition. Eur J Clin Microbiol Infect Dis 2009;28:989-90.

9. Dhillon RH, Clark J. ESBLs: A Clear and Present Danger? Crit Care Res Pract 2012;2012:625170. Published online 6 June 2011. [PMC free articale] [PubMed].

10. Das N, Borthakur AK. Antibiotic coresistance among extended-spectrum beta lactamase-producing urinary isolates in a tertiary medical center: A prospective study. Chronicles of Young Scientists Chron Young Sci. 2012;3:53-6.

11. Rudresh SM, Nagarathnamma T. Extended spectrum $\quad \beta$-lactamase producing Enterobacteriaceae and antibiotic coresistance. Indian J Med Res 2011;133:116-8.

12. Umadevi S, Kandhakumari G, Joseph NM, Kumar S, Easow JM, Stephen S, et al. Prevalence and antimicrobial susceptibility patt ern of ESBL producing Gram Negative Bacilli. J Clin Diagn Res 2011;5:236-9.

13. Metri Basavaraj C, Jyothi P, Peerapur Basavaraj V. The prevalence of ESBL among Enterobacteriaceae in a tertiary care hospital of North Karnataka, India. J Clin Diagn Res 2011;5:470-5

14. Kumar MS, Lakshmi V, Rajagopalan R. Occurrence of extended spectrum betalactamases among Enterobacteriaceae spp. isolated at a tertiary care institute. Indian $\mathbf{J}$ Med Microbiol 2006;24:208-11

15. Rodrigues C, Joshi P, Jani SH, Alphonse M, Radhakrishnan R,Mehta A. Detection of $\square$-Lactamases in nosocomial Gram 
negative clinical isolates. Indian $\mathrm{J}$ Med Microbiol 2004;22:247-250.

16. Collee JG, Fraser AG, Marmian BP, Simmons A, editors. Mackie and McCartney Practical Medical Microbiology. $14^{\text {th }}$ ed. Standard ed. Churchill Livingstone, London,1996. (Reprinted 1999.

17. Win WC, Allen SD, Janda WM, Koneman EW, Procop GW,Schreckenberger PC, Woods G, editors. Colour atlas and text book of diagnostic microbiology. 6th ed. Philadelphia: Lippincott Williams

and Wilkins; Enterobacteriaceae; 2006. p. 211302.

18. Clinical Laboratory Standards Institute. Performance Standard for Antimicrobial Susceptibility Testing. $23{ }^{\text {rd }}$ Information Supplement. NCCLS Document M100S23. Wayne, PA, USA, 2017

19. Mohamudha PR, Harish BN, Parij a SC. Amp $\mathrm{C}$ beta lactamases among gram negative clinical isolates from a tertiary hospital,South India. Braz J Microbiol 2010;41:596-602.

20. Polsfuss S, Bloemberg GV, Giger J, Meyer $\mathrm{V}$, Bott ger EC,Hambach M. Practical approach for reliable detection of AmpC beta-lactamases

Enterobacteriaceae. J Clin Microbiol

2011;49:2798-803.

21. Sharma M. Prevalence and antibiogram of Extended Spectrum $\beta$-Lactamase (ESBL) producing Gram negative bacilli and further molecular characterization of ESBL producing Escherichia coli and Klebsiella spp. Journal of Clinical and Diagnostic Research. 2013;7(10):2173-77.

22. Mendelson G, Hait V, Ben-Israel J, Gronich D, Granot E, Raz R. Prevalence and risk factors of extended-spectrum betalactamase-producing Escherichia coli and Klebsiella pneumoniae in an Israeli longterm care facility. European Journal of Clinical Microbiology \& Infectious Diseases. 2004;24(1):17-22.
23. Bazzaz B, Naderinasab M, Mohamadpoor A, Farshadzadeh Z, Ahmadi S, Yousefi F. The prevalence of extended-spectrum betalactamase-producing Escherichia coli and Klebsiella pneumoniae among clinical isolates from a general hospital in Iran. Acta Microbiologica et Immunologica Hungarica. 2009;56(1):89-99.

24. Kiratisin P, Apisarnthanarak A, Laesripa C, and Saifon P. Molecular characterization and epidemiology of extended-spectrum $\beta$-lactamaseproducing Escherichia coli and Klebsiella pneumoniae isolates causing health careassociated infection in Thailand, where the CTX-M family

is endemic. Antimicrob Agents Chemother 2008;52(8):2818-24.

25. Basavaraj C.Metri et al.The Prevalence of ESBL among Enterobactericeae in a Tertiary Care Hospital of North Karnataka, India.JCDR 2011 June,Vol-5(3):470-475.

26. Mathur P, Kapil A, Das B, Dhawan B. Prevalence of extended spectrum $\beta$ lactamase producing Gram negative bacteria in tertiary care hosptital. Indian $J$ Med Res 2002;115:153-157.

27. Babypadmini S, Appalaraju B. Extended spectrum $\beta$-lactamases in urinary isolates of Escherichia coli and Klebsiella pneumoniae -Prevalence and susceptibility pattern in a tertiary care hospital. Indian $J$ Med Microbiol 2004;22(3):172-174.

28. Sasirekha B. Prevalence of ESBL, AMPC B-lactamases and MRSA among uropathogens and its antibiogram. EXCLI J 2013;12:81-8.

29. Ndugulile F, Jureen R, Harthug S, Urassa W, Langeland N. Extended spectrum betalactamases among Gram-negative bacteria of nosocomial origin from an intensive care unit of a tertiary health facility in Tanzania. BMC Infect Dis 2005;5:86.

30. Mehrgan H, Rahbar M, Arab-Halvaii Z. High prevalence of extended-spectrum beta-lactamase-producing Klebsiella 
pneumoniae in a tertiary care hospital in Tehran, Iran. J Infect Dev Ctries 2010;4:132-8.

31. Paterson DL. Resistance in gram-negative bacteria: Enterobacteriaceae. Am J Infect Control 2006;34:20-8.

32. Rodríguez-Martínez JM, Poirel L, Nordmann P. Molecular epidemiology and mechanisms of carbapenem resistance in Pseudomonas aeruginosa. Antimicrob Agents Chemother 2009;53:4783-8.

33. Aruna K, Mobashshera T. Prevalence of extended spectrum beta-lactamase production among uropathogens in South
Mumbai and its antibiogram patterns. EXCLI J 2012;11:363-72.

34. Sasirekha B, Shivakumar S. Occurrence of plasmid-mediated AmpC $\beta$-lactamases among Escherichia coli and Klebsiella pneumoniae clinical isolates in a tertiary care hospital in Bangalore. Indian $\mathbf{J}$ Microbiol 2012;52:174-9.

35. Gupta E, Mohanty S, Sood S, Dhawan B, Das BK, Kapil A. Emerging resistance to carbapenems in a tertiary care hospital in north India. Indian J Med Res 2006; $124: 95-8$.

\section{How to cite this article:}

Sanjo Gupta and Veena Maheshwari. 2017. Prevalence of ESBLs among Enterobacteriaceae and their Antibiotic Resistance Pattern from Various Clinical Samples. Int.J.Curr.Microbiol.App.Sci. 6(9): 2620-2628. doi: https://doi.org/10.20546/ijcmas.2017.609.323 